\title{
Hybrid Methodology for Efficient on the Fly (Re)Parametrization of Proton Exchange Membrane Fuel Cells Electrochemical Model for Diagnostics and Control Applications
}

\author{
A. $\operatorname{Kravos}^{\mathrm{a}}$, A.Kregara, ${ }^{\mathrm{a}, \mathrm{b}}$, and T. Katrašnik ${ }^{\mathrm{a}}$ \\ ${ }^{a}$ University of Ljubljana, Faculty of Mechanical Engineering, Aškerčeva 6, SI-1000 \\ Ljubljana, Slovenia \\ ${ }^{\mathrm{b}}$ University of Ljubljana, Faculty of Education, Kardeljeva ploščad 16, SI-1000 \\ Ljubljana, Slovenia
}

\begin{abstract}
Successful parametrization and re-parametrization of the models used in PEMFC observer applications is instrumental to assure ideal control of the system. To increase ease of parametrisation this paper presents framework of twin analytical ansatzes for modelling PEMFC response in time and frequency domains sharing same set of calibration parameters. Owing to thermodynamically consistent modelling basis of the twin models, which are based on electrochemical model with state-of-the-art extrapolation capabilities and replication of the experimental data with one set of calibration parameters, they are valid in all current density regions. Furthermore, unique sharing of the calibration parameters enables unprecedented enrichment of the dataset that can be used to determine values of model's calibration parameters with higher certainty or enhances identification of individual calibration parameters that are otherwise harder to be uniquely determined. Additionally, proposed hybrid methodology also enables a significant reduction in the measurement time and enable reparametrization on the fly.
\end{abstract}

\section{Introduction}

Prevention and diminution of detrimental degradation phenomena under highly dynamic operation of proton exchange membrane fuel cells (PEMFC), while retaining sufficiently high efficiency and performance of the system, are considered significant obstacles toward their wider use in transport applications. Overcoming these obstacles calls for precise online monitoring and control tools such as coupled observers, which enable combined performance and service life optimizations. Models used in these applications should feature low computational effort, good extrapolation capabilities and should be easy to parametrize. The first requirement, which is obvious for on-line applications, in frequently in contradiction to the last two, which are heavily intertwined. Good extrapolation capabilities of the model significantly reduce the size of experimental data sets needed for successful parametrization, thus enabling the parametrization on small data sets, while retaining higher accuracy outside of trained variation space. This rationale motivates the use of the computationally-fast reduced-dimensionality electrochemical models e.g. $(1,2,3)$,

This Author Accepted Manuscript (AAM) is published in:

ECS Transactions, Volume 98, Number 9, 13-24, 2020

DOI: 10.1149/09809.0013ecst 
featuring a more profound mechanistic basis; thus, exhibiting better prediction and thus also extrapolation capability of the model.

Development and validation process of the thermodynamically consistent reduced dimensionality electrochemical model (TC FC electrochemical model) (2), used to simulate response of PEMFC time domain, showed that identifiability of individual calibration parameters and correct parametrization of the model represent crucial steps for successful utilization of this and also of any other system level models. Sufficient identifiability of individual calibration parameters can be assured with the reduction of the model (2) or by using suitable experimental data in parametrization procedure (4). Reduction of the model can be a viable option in cases, when calibration parameters exhibit mathematically confirmed inter-correlations or interdependence and should be in other cases avoided since it reduces parameter dimensionality of the model and thus, hinders extrapolation capabilities and physical significance of the model. Furthermore, it has been shown (4), that even though some calibration parameters do not exhibit any interdependencies, they still cannot be uniquely determined based on limited amount of experimental data obtained in time domain. Increasing amount of suitable experimental data would increase information about individual parameters to some extent, however availability of experimental data is limited by time and cost constraints. In addition, some calibration parameters e.g. the ones describing anodic and cathodic reaction rates and their activation energies are extremely hard to be uniquely determined based on the experimental data consisting of polarization curves in normal operating conditions only. This inherently means that unique identifiability of the calibration parameters, based solely on time domain experimental data, is very challenging without reduction of the time domain model. This problem is even more pronounced for fuel cell stacks integrated in series production FC systems, where control system and $\mathrm{BoP}$ characteristics further limit the size of variation space thus further limiting unique determination of model parameters. Since excess reduction of the time domain model should be avoided to preserve its generality and physical significance, innovative approaches need to be developed.

With an aim of successfully tackling this problem of unique calibration parameter indentifiability, this contribution presents framework of computationally-fast electrochemical models that can be parametrized either on experimental data from time domain (with e.g. polarization curves) and/or on data obtained in frequency domain (by electrochemical impedance spectroscopy (EIS)).

Originating from the aforementioned required features of electrochemical models used for virtual observer parametrization, it can be concluded that data driven models in the frequency domain, which are often applied in the system level analyses, have limited applicability for modelling of EIS spectra in studies with an objective of combined performance and service life optimisations. This is reasoned by the fact that the accuracy of data driven models generally, does not reach beyond the trained variation space of parameters and due to the fact that training data driven models on a variation space with a very large dimensionality proves to be cumbersome due to the effects of i.e. the curse of dimensionality (5). This reasoning is fully applicable in case of equivalent circuit models such as the ones proposed in e.g. $(6,7,8)$. An advance in this area was made by Eikerling (9), Niya (10) and Kulikovsky (11), however all of them used Tafel approximation for

This Author Accepted Manuscript (AAM) is published in:

ECS Transactions, Volume 98, Number 9, 13-24, 2020

DOI: 10.1149/09809.0013ecst 
calculation of the current density. This approximation is a reasonable for operational points with high current densities and consequential high activation overvoltages. However, it possesses a significant downside in the low current density region, where the approximation error increases exponentially, when current density approaches zero, which inevitably means that the activation losses cannot be well defined.

To efficiently address the ambitious objective of parametrization of TC FC electrochemical model with data measured in the time and in the frequency domain, while additionally eliminating limitations of applying EIS model at low current densities due to Tafel approximation, this paper presents an innovative hybrid methodology for efficient on the fly re-parametrization of PEMFC electrochemical model. This methodology relies on TC FC electrochemical model presented in (2), which is demonstrated to be highly accurate when calibrated at multiple polarization curves at once and to have state-of-the-art extrapolation capabilities outside trained variation space (2). Innovative extension presented in this paper focuses on derivation of the model twin in the frequency domain, by using proposed modelling approach for calculation of the cathode side of a PEMFC impedance in the inspiring work of Kulikovsky (11). Derived model is based on analytical solution of the PEMFC impedance and is valid over all current density regions due to more profound modeling ansatzes from (2). Furthermore, it comprises all calibration parameters of the time domain model and two additional parameters. This unique sharing of the calibration parameters enables unprecedented enrichment of the dataset that can be used to determine values of model's calibration parameters with higher certainty or enhances identification of individual calibration parameters that are otherwise harder to be uniquely determined (4). This is also very important for achieving faster calibration times with higher repeatability. Furthermore, proposed hybrid methodology also enables a significant reduction in the measurement time in the case of experimental data obtained by EIS, allowing the low frequency regions, where the transport phenomena could be otherwise characterized, to be omitted due to already uniquely determined calibration parameters from polarization curve measurements. Moreover, hybrid parametrization is even more important for on-line application and state of $\mathrm{X}(\mathrm{SoX})$ monitoring. During on-line operation it is in general not possible to measure the required dataset in time domain to properly reparametrize the model with the aim to detect effects of degradation phenomena or malfunctioning. However, predictions of the model in the time domain are crucial to properly predict SoF (State of Function) and variation in SoH (State of Health) for specific operation. In this cases re-parametrization with the data obtained in the frequency domain allows for unprecedented on the fly re-parametrization of the model with higher certainty. Innovative hybrid methodology of the measurements in time and frequency domains could thus be used for achieving reduced costs and efforts in initial parametrization and during operation in re-parametrization procedures of the modelling framework for virtual observers.

To consistently address presented problem, the paper is structured as following, firstly Modelling framework section briefly presents the governing equations of Time domain FC electrochemical model that are relevant for optimization procedure and derivation of frequency domain FC electrochemical model twin. The model is derived and solved analytically for small amplitude EIS excitations applying harmonic linearization step to governing PDEs. In section Results, results of calibration on the multiple polarization 
curves is presented and obtained calibration parameters are used to successfully parametrize the newly developed physical model for impedance spectra. In the last section main conclusions are summarized.

\section{Modelling framework}

\section{$\underline{\text { Time domain FC electrochemical model }}$}

Time domain FC electrochemical model exhibits state-of-the-art extrapolation and interpolation capabilities for operational points outside of the trained variation space. Furthermore, it enables ease of parametrization on the small data sets and exhibit small root mean square deviation when compared against multiple polarization curves calibrated at once. This state of the art model properties are a result of mathematically consistent use of the sinus hyperbolicus definition on the Butler-Volmer (BV) equation. This approach results in an equation equivalent to the $\mathrm{BV}$ and is valid over all current density ranges. Detailed analysis of this properties and the derivation of the model is presented in the scope of (2). The model is in the scope of aforementioned work further extended to accommodate the transport of gaseous species along the channel and through the gas diffusion layer (GDL) yielding quasi-one-dimensional model. Within modelling framework for hybrid methodology it is used to model response of PEMFC in time domain. The main governing equation can be written as:

$$
\begin{gathered}
U= \\
\frac{k_{b} T}{e_{0} Z_{c}} \ln \left(\left(\tilde{C}_{H_{2} O}\left(1+\frac{I}{I_{L_{c}}}\right)\right)^{-2}\left(\tilde{C}_{O_{2}}\left(1-\frac{I}{I_{L_{c}}}\right)\right)-\frac{k_{b} T}{e_{0} z_{c}} \ln \left(\left(\tilde{C}_{H_{2}}\left(1-\frac{I}{I_{L_{a}}}\right)\right)^{2}\right)+\right. \\
+\frac{\Delta g_{c}^{0}}{e_{0} Z_{c}}+\frac{\left(T-T^{0}\right) \Delta s_{C}}{e_{0} Z_{c}}+\frac{\Delta g_{a}^{0}}{e_{0} Z_{a}}+\frac{\left(T-T^{0}\right) \Delta s_{a}}{e_{0} Z_{a}}-R I+ \\
-\frac{k_{b} T}{e_{0} Z_{c}} \sinh ^{-1}\left(\frac{I}{2 \widetilde{I_{c}^{0}}}\left(\tilde{C}_{H_{2} O}\left(1+\frac{I}{I_{L_{c}}}\right)\right)^{-1}\left(\tilde{C}_{O_{2}}\left(1-\frac{I}{I_{L_{c}}}\right)\right)^{-0.5}\right)+ \\
-\frac{k_{b} T}{e_{0} Z_{a}} \sinh ^{-1}\left(\frac{I}{2 \widetilde{I}_{a}^{0}}\left(\tilde{C}_{H_{2}}\left(1-\frac{I}{I_{L_{a}}}\right)\right)^{-1}\right)
\end{gathered}
$$

where $k_{b}$ is the Boltzmann constant, $T$ is the temperature, $e_{0}$ is the elementary charge, $\Delta g_{a}^{0}$ and $\Delta g_{c}^{0}$ are the differences in specific Gibbs free energy between reactants and products on the anode and cathode side and similarly $\Delta s_{a}$ and $\Delta s_{c}$ are the specific entropy differences on the anode and cathode side, $Z_{a}$ and $Z_{c}$ are the number of electrons transferred in the electrochemical reaction on the anode and cathode side, values of $\tilde{C}_{\mathrm{H}_{2} \mathrm{O}}$, $\tilde{C}_{\mathrm{O}_{2}}$ and $\tilde{C}_{\mathrm{H}_{2}}$ represent normalized values of concentrations of water, oxygen and hydrogen, respectively. $R$ is the resistance, $\widetilde{I_{a}^{0}}$ and $\widetilde{I_{c}^{0}}$ are intrinsic exchange current density on the anode and cathode side and can be defined as: 


$$
\widetilde{I_{c / a}^{0}}=Z F S \cdot \mathrm{e}^{-\frac{\alpha_{c / a^{\Delta} \Delta s_{c / a}\left(T-T^{0}\right)}^{0}}{k_{b} T}} I_{c / a}^{0}
$$

where $F$ is Faraday constant and $S$ is active area of FC. For the anode side expression is analogous. On the other hand $I_{L_{a}}$ and $I_{L_{c}}$ are limiting currents which are defined as:

$$
I_{L_{c / a}}=Z F S D_{c / a} \frac{C_{r_{c h} / a}}{\delta_{G D L_{c / a}}}=Z F S C_{r_{c h_{c / a}}} C D_{c / a}
$$

with $C_{r_{c h} / a}$ being the concentration of reactants in the channel and $D_{c / a}$ being the effective diffusivity through the GDL on the anode and cathode side. To determine a set of calibration parameters, firstly inputs based on the operational conditions and known physical constants, were inserted into the model. The remaining parameters presented the set of calibration parameters: $I_{a}^{0}, I_{c}^{0}, R, C D_{a}$ and $C D_{c}$.

Frequency domain twin of the FC model

The model for catalyst layer (CL) performance has been studied in detail in $(12,13,14)$, where following system of equations was devised for 1D model in $\mathrm{x}$-direction, which is perpendicular to the CL:

$$
\begin{gathered}
\mathrm{C}_{\mathrm{DL}} \frac{\partial \eta(x, t)}{\partial t}+\frac{\partial j(x, t)}{\partial x}=-S(x, t), \\
j(x, t)=-\sigma \frac{\partial \eta(x, t)}{\partial x}, \\
\frac{\partial \tilde{C}(x, t)}{\partial t}-D_{e f f} \frac{\partial^{2} \tilde{C}(x, t)}{\partial x^{2}}=-\frac{S(x, t)}{Z F},
\end{gathered}
$$

where, $\mathrm{C}_{\mathrm{DL}}$ is double layer capacitance of $\mathrm{CL}, \eta(x, t)$ is overpotential, $j(x, t)$ is ionic current, $\sigma$ is ionic conductivity of the catalyst layer, $\tilde{C}(x, t)$ is oxygen concentration in CL, $D_{\text {eff }}$ is effective diffusivity of the oxygen in the GDL, and $S(x, t)$ is a source term which was derived in detail for time domain model (2) and can be written as:

$$
S(x, t)=\widetilde{I_{c}^{0}} \tilde{C}_{O_{2}}^{0.5}(x, t) \tilde{C}_{H_{2} O} 2 \sinh \left(\frac{e_{0} Z \eta(x, t)}{k_{b} T}\right)
$$


where $\widetilde{I_{c}^{0}}$ is intrinsic exchange current density defined with equation [2]. It is worth noticing that concentration of water is, with an aim of obtaining analytical solution assumed constant throughout CL. Additionally, equation [7] can be written also as:

$$
S(x, t)=\zeta \tilde{C}_{O_{2}}^{0.5}(x, t) \sinh (\gamma \eta(x, t)),
$$

where $\zeta$ and $\gamma$ are intrinsic constants. Therefore, the set of governing equations consistent with the time domain model can be written in full form as:

$$
\begin{gathered}
\mathrm{C}_{\mathrm{DL}} \frac{\partial \eta(x, t)}{\partial t}+\frac{\partial j(x, t)}{\partial x}=-\zeta \tilde{C}(x, t)^{0.5} \sinh (\gamma \eta(x, t)), \\
j(x, t)=-\sigma \frac{\partial \eta(x, t)}{\partial x}, \\
\frac{\partial \tilde{C}(x, t)}{\partial t}-D_{e f f} \frac{\partial^{2} \tilde{C}(x, t)}{\partial x^{2}}=-\frac{\zeta}{Z F} \tilde{C}(x, t)^{0.5} \sinh (\gamma \eta(x, t)), \\
\frac{\partial \tilde{C}_{G D L}(x, t)}{\partial t}-D_{e f f_{G D L}} \frac{\partial^{2} \tilde{C}_{G D L}(x, t)}{\partial x^{2}}=0,
\end{gathered}
$$

where, additional equation [12] describing diffusive oxygen transport is presented, with $\widetilde{\boldsymbol{C}}_{G D L}(\boldsymbol{x}, \boldsymbol{t})$ being concentration of oxygen in GDL, and $\boldsymbol{D}_{\boldsymbol{e f f} f_{G D}}$ effective diffusion constant for oxygen in GDL.

Obtained set of governing equations describes the performance characteristics of individual electrode. With an aim of obtaining impedance of individual electrode, small harmonic perturbations have to be applied on the overpotential, concentration of oxygen in GDL and CL. The latter can be written as summation of steady-state solution and small harmonic perturbation, such as:

$$
\begin{aligned}
& \eta=\eta_{\mathrm{S}}+\eta_{D} e^{-i \omega t}, \\
& C=C_{S}+C_{D} e^{-i \omega t}, \\
& C_{G D L}=C_{S_{G D L}}+C_{D_{G D L}} e^{-i \omega t},
\end{aligned}
$$

where, $\boldsymbol{\eta}_{\mathrm{S},} \boldsymbol{C}_{\boldsymbol{S}}, \boldsymbol{C}_{\boldsymbol{S}_{G D L}}$ represent the steady-state solution, $\boldsymbol{\eta}_{\boldsymbol{D}}, \boldsymbol{C}_{\boldsymbol{D}}, \boldsymbol{C}_{\boldsymbol{D}_{G D L}}$ represent the amplitude of perturbation and $\boldsymbol{\omega}$ is circular frequency. For the sake of brevity only derivation on the cathode side will be presented. It should be pointed out that modelling approach based on system of equations $[4-6,12]$ was used for calculation of the cathode catalyst layer impedance in the inspiring work of Kulikovsky (11). However, instead of 
equivalent of BV equation as proposed in (2) and used in the time domain model the source term in (11) was defined based on the Tafel description of kinetics which is a reasonable approximation for operational points with high current densities and consequential high activation overvoltages. The drawback of this approach arises from the fact that it possesses a significant downside in the low current density region, where the approximation error increases exponentially when current density approaches zero, which inevitably means that the activation losses cannot be well defined. This difference in source ansatzes leads to different derivation results in (11), where analytical solution is valid only for small current impedance, whereas governing equations derived in the scope of this paper are valid for all current density regions.

Inserting equations [13] into the system of equations [9-12] and applying standard harmonic linearization procedure on them leads to a new set of governing equations for perturbation amplitudes:

$$
\begin{gathered}
\frac{\partial^{2} \eta_{D}}{\partial^{2} x}=R \eta_{D}+S \tilde{C}(x, t)^{0.5}, \\
\frac{\partial^{2} \tilde{C}(x, t)}{\partial x^{2}}=U \eta_{D}+W \tilde{C}(x, t)^{0.5}, \\
\frac{\partial^{2} \tilde{C}_{G D L}(x, t)}{\partial x^{2}}=V \tilde{C}_{G D L}(x, t),
\end{gathered}
$$

with, $R, S, U, W$, and $V$ being:

$$
\begin{gathered}
R=\frac{\left(i \omega C_{D L}+\gamma \zeta c_{s}^{0.5} \cosh \left(\eta_{s} \gamma\right)\right)}{\sigma} ; \\
S=\frac{\zeta \sinh \eta_{s} \gamma}{\sigma} ; \\
W=\frac{\zeta}{2 Z_{c} F} c_{s}^{0.5} \cosh \left(\eta_{s} \gamma\right) \frac{\gamma}{D_{e f f}} ; \\
V=\frac{i \omega+\frac{\zeta}{2 Z_{c} F} \sinh \eta_{s} \gamma}{D_{e f f}} \\
D_{e f f_{G D L}}
\end{gathered}
$$

Boundary conditions. Set of coupled equations presented in equations [14-16] can be solved for proper boundary conditions, With an aim of easier representation non- 
dimensional length $\tilde{\mathrm{x}}=\frac{x}{\delta_{C L}}$ is introduced. Boundary conditions shown in equation [18] are representation of the following physical phenomenon:

- At interface between GDL and CL $(\tilde{\mathrm{x}}=1)$ concentration and flux are continuous

- At membrane CL interface $(\widetilde{\mathrm{x}}=0)$, oxygen flux is zero

- At interface between GDL and CL $(\tilde{\mathrm{x}}=1)$ there is known overpotential and zero proton current.

- At GDL channel interface $\left.\widetilde{(\mathrm{x}}=1+\delta_{G D L} / \delta_{C C L}\right)$ concentration is the same as the one in the channel.

$$
\begin{aligned}
& \frac{\partial \tilde{C}(\tilde{\mathrm{x}}=0, \mathrm{t})}{\partial \tilde{\mathrm{x}}}=0 ; \tilde{C} Y Z(\tilde{x}=1, t)=\tilde{C}_{G D L}(\tilde{x}=1, t) \\
& D_{e f f} \frac{\partial \tilde{C}(\tilde{\mathrm{x}}=1, t)}{\partial x}=D_{e f f_{G D L}} \frac{\partial \tilde{C}_{G D L}(\tilde{\mathrm{x}}=1, t)}{\partial x} ; \frac{\partial \eta(\tilde{\mathrm{x}}=1, \mathrm{t})}{\partial \tilde{\mathrm{x}}}=0 ; \\
& \eta(\tilde{\mathrm{x}}=1, \mathrm{t})=\eta_{D} ; \tilde{C}_{G D L}\left(\tilde{\mathrm{x}}=1+\delta_{G D L} / \delta_{C L}, t\right)=\tilde{C}_{c h}(t) ;
\end{aligned}
$$

with $\boldsymbol{\delta}_{\boldsymbol{G D \boldsymbol { L }}}$ and $\boldsymbol{\delta}_{\boldsymbol{C} \boldsymbol{L}}$, being widths of GDL and CL respectively, and $\widetilde{\boldsymbol{C}}_{\boldsymbol{c h}}$ being concentration in the channel. The solution of the coupled governing equations is in the scope of his paper shown only for $\boldsymbol{\eta}_{\boldsymbol{D}}$, however analytical solutions for concentrations profiles can be obtained as well. Solution for overpotential profile is sought, due to the fact that the impedance of the system $\check{\mathbf{Z}}$, can be given by:

$$
\check{\mathrm{Z}}=-\frac{\eta_{D}(\tilde{x}=0)}{\frac{\partial \eta_{D}(\tilde{x}=0)}{\partial \tilde{x}}}
$$

Obtained analytical solution is long and cumbersome, however if few additional characteristic constants are defined as:

$$
\begin{gathered}
X=\sqrt{\left(R+\sqrt{\left(4 * S * U+(R-W)^{2}\right)}+W\right)} ; \\
Y=\sqrt{\left(R-\sqrt{\left(4 * S * U+(R-W)^{2}\right)}+W\right)} ; \\
Z=\sqrt{(-S * U+R * W)} ; \\
Q=\sqrt{\left(4 * S * U+(R-W)^{2}\right)} ;
\end{gathered}
$$




$$
\begin{gathered}
\phi=\eta_{\mathrm{D}}\left(R S U Y+S U(Q+W) Y+R^{2} X Z+2 S U X Z+W X(W Z-\mathrm{ZQ})+R X(-2 W Z+\mathrm{ZQ})\right) \\
-\frac{\mathrm{C}_{\mathrm{c}}^{\mathrm{c}} S\left(2 S U Y+Q W Y-R W Y+W^{2} Y+R X Z-W X Z+X \mathrm{ZQ}\right) \mathrm{Tanh}\left[\frac{\delta_{G D L}}{\delta_{C C L}} \sqrt{V}\right]}{\sqrt{V}} \\
\chi=\eta_{\mathrm{D}}\left(R S U X+S U(-Q+W) X+R^{2} Y Z+2 S U Y Z+W Y(W Z+\mathrm{ZQ})-R Y(2 W Z+\mathrm{ZQ})\right) \\
+\frac{\mathrm{C}_{\mathrm{c}}^{\mathrm{c}} S\left(-2 S U X+Q W X+R W X-W^{2} X-R Y Z+W Y Z+Y Z Q\right) \operatorname{Tanh}\left[\frac{\delta_{G D L}}{\delta_{C C L}} \sqrt{V}\right]}{\sqrt{V}} \\
\psi=2 Z \operatorname{Cosh}\left[\frac{X}{\sqrt{2}}\right] \operatorname{Cosh}\left[\frac{Y}{\sqrt{2}}\right]\left(\eta_{\mathrm{D}}\left(2 S U+(R-W)^{2}\right)+\frac{\mathrm{C}_{\mathrm{c}}^{\mathrm{c}} S(-R+W) \operatorname{Tanh}\left[\frac{\delta_{G D L}}{\delta_{C C L}} \sqrt{V}\right]}{\sqrt{V}}\right) \\
\xi=-\sqrt{2} Q \mathrm{C}_{\mathrm{c}}^{\mathrm{c}} \sqrt{-Q+R+W} \operatorname{Sinh}\left[\frac{X}{\sqrt{2}}\right]+2 Z\left(2 \eta_{\mathrm{D}} U+\frac{\mathrm{C}_{\mathrm{c}}^{\mathrm{c}}(R-W) \operatorname{Tanh}\left[\frac{\delta_{G D L}}{\delta_{C C L}} \sqrt{V}\right]}{\sqrt{V}}\right) \\
\mu=\frac{2 \operatorname{Sinh}\left[\frac{X}{\sqrt{2}}\right]\left(\eta_{D} U \sqrt{V}(R+W)-\mathrm{C}_{\mathrm{c}}^{\mathrm{c}}\left(2 S U-R W+W^{2}\right) \operatorname{Tanh}\left[\frac{\delta_{G D L}}{\delta_{C C L}} \sqrt{V}\right]\right)}{\sqrt{V}}
\end{gathered}
$$

Equation reduces in length and gives the system impedance on the cathode side defined as:

$$
\begin{gathered}
\check{\mathrm{Z}}_{\mathrm{c}}=- \\
\frac{2 \delta_{C L}\left(\psi+S\left(\xi+\operatorname{Sinh}\left[\frac{Y}{\sqrt{2}}\right]\left(\sqrt{2} \mathrm{C}_{\mathrm{c}}^{\mathrm{c}} Q \sqrt{Q+R+W}+\mu\right)\right)\right)}{\operatorname{Cosh}\left[\frac{Y}{\sqrt{2}}\right]\left(2 \mathrm{C}_{\mathrm{c}}^{\mathrm{c}} Q S \sqrt{Q+R+W} Y+\sqrt{2} \phi \operatorname{Sinh}\left[\frac{X}{\sqrt{2}}\right]\right)+\operatorname{Cosh}\left[\frac{X}{\sqrt{2}}\right]\left(-2 \mathrm{C}_{\mathrm{c}}^{\mathrm{c}} Q S \sqrt{-Q+R+W} X+\sqrt{2} \chi \operatorname{Sinh}\left[\frac{Y}{\sqrt{2}}\right]\right)}
\end{gathered}
$$

Similar solution can be obtained also for the anode side, therefore the whole impedance of the PEMFC can be written as summation of impedance of cathode and anode $\left(\widetilde{\mathbf{Z}_{\mathbf{c}}}\right.$ and $\left.\widetilde{\mathbf{Z}_{\mathbf{a}}}\right)$ side and contribution of the membrane impedance $(\boldsymbol{R})$, which is currently only a scalar resistance value.

$$
\overrightarrow{\mathrm{Z}}=\breve{\mathrm{Z}}_{a}+\breve{\mathrm{Z}}_{c}+\vec{R}
$$

In the next step the determination of set of calibration parameters was determined. After inserting inputs based on the operational conditions and known physical constants, into the newly derived model. The remaining unknown parameters presented the set of calibration parameters, that consisted of: $\boldsymbol{I}_{\boldsymbol{a}}^{\mathbf{0}}, \boldsymbol{I}_{\boldsymbol{c}}^{\mathbf{0}}, \boldsymbol{R}, \boldsymbol{C} \boldsymbol{D}_{\boldsymbol{a}}, \boldsymbol{C} \boldsymbol{D}_{\boldsymbol{c}}, \boldsymbol{C}_{\boldsymbol{D L}}$ and $\boldsymbol{\sigma}$; thus representing the extended set of calibration parameters used in time domain of FC electrochemical model by double layer capacitance and proton conductivity values.

\section{This Author Accepted Manuscript (AAM) is published in:}

ECS Transactions, Volume 98, Number 9, 13-24, 2020

DOI: 10.1149/09809.0013ecst 


\section{Hybrid methodology}

Hybrid methodology revolves around the twin electrochemical models presented in equation [1] for time domain and [22] for frequency domain. The core set of calibration parameters for both models is interchangeable and it consists of $\boldsymbol{I}_{\boldsymbol{a}}^{\mathbf{0}}, \boldsymbol{I}_{\boldsymbol{c}}^{\mathbf{0}}, \boldsymbol{R}, \boldsymbol{C} \boldsymbol{D}_{\boldsymbol{a}}, \boldsymbol{C} \boldsymbol{D}_{\boldsymbol{c}}$. This unique sharing of the calibration parameters enables unprecedented enrichment of the dataset for parametrization and enables parametrization from time or frequency domain independently.

Both of the models are devised in a manner that can be interchangeably plugged into optimization tool that is revolved around differential evolution algorithm and NelderMead optimization algorithm. Differential evolution is metaheuristic evolutionary optimization algorithm that does not need the optimization problem to be differentiable to optimize it, which inherently means that it is less prone to getting stuck in local minimum. Moreover, the optimization is also possible on experimental data from both domains at the same time with the same or different "weights" applied in optimization function for each of the domains.

\section{Results}

The modelling framework devised in previous sections was utilized on the experimental data obtained in time domain, which consisted of four individual polarization curves and which were obtained on the FC stack at different operating conditions as defined in Table 1.

TABLE I. Operating conditions of FC under which polarisation curves were obtained.

\begin{tabular}{cccccccc}
\hline No. & $\boldsymbol{\lambda}_{\text {an }}$ & $\boldsymbol{\lambda}_{\text {cat }}$ & p $_{\text {in.an[bar] }}$ & pin.cat[bar] & $\mathbf{R H}_{\text {an[ }[\%]}$ & $\mathbf{R H}_{\text {cat[\%] }}$ & $\mathbf{T}[\mathbf{K}]$ \\
\hline 1 & 1.3 & 1.8 & 1.013 & 1.013 & 0 & 80 & 343.15 \\
2 & 1.3 & 1.7 & 1.4 & 1.4 & 0 & 80 & 346.15 \\
3 & 1.3 & 1.7 & 2.0 & 2.0 & 0 & 80 & 347.15 \\
4 & 1.3 & 1.7 & 2.5 & 2.5 & 0 & 80 & 349.15 \\
\hline
\end{tabular}

Calibration of the model was performed via minimalisation of the penalty function values, which in this case meant reducing root square deviation of model output from experimental data. Furthermore, to assure physically plausible results, value constraints were introduced on calibration parameters. The calibration process was then run with differential evolution. The results obtained with the calibrated TC FC electrochemical model are shown in Figure 1 and exhibit good correlation between experiment and model output with RMSD value of 0.00561 . 


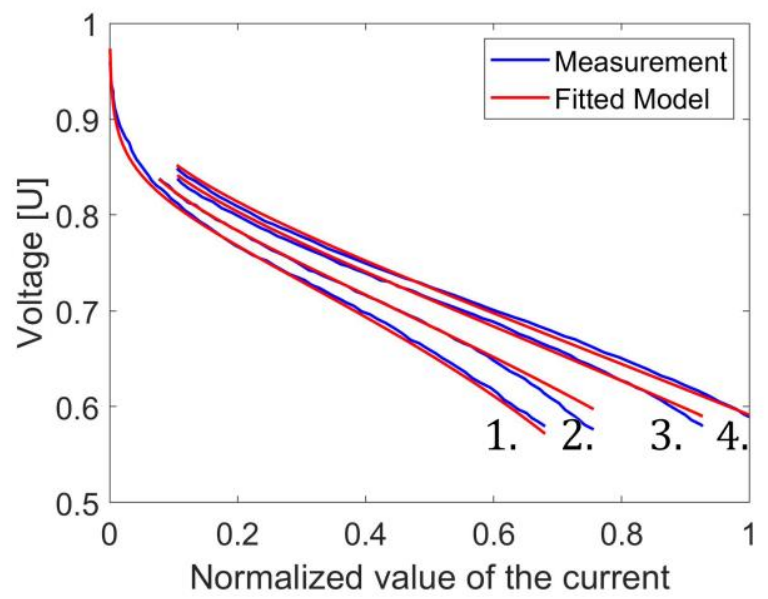

Figure 1: Results of the calibrated model for multiple polarisation curves fitted at once.

Calibration parameters obtained with this optimization process were then, as described in section Hybrid methodology, utilized in the newly derived frequency domain twin of the FC model impedance spectra together with literature known values of double layer capacitance $\left(22 \mathrm{Fcm}^{-3}(11)\right)$ and proton conductivity $\left(0.0582 \Omega^{-1} \mathrm{~cm}^{-1}(11)\right)$, which would, in case of the experimental data that consists of EIS spectra, be optimized as calibration parameters. Results presented in Figure 2, shown meaningful results throughout the whole frequency range, furthermore it has also detailed high frequency which enables precise determination of ohmic resistance.

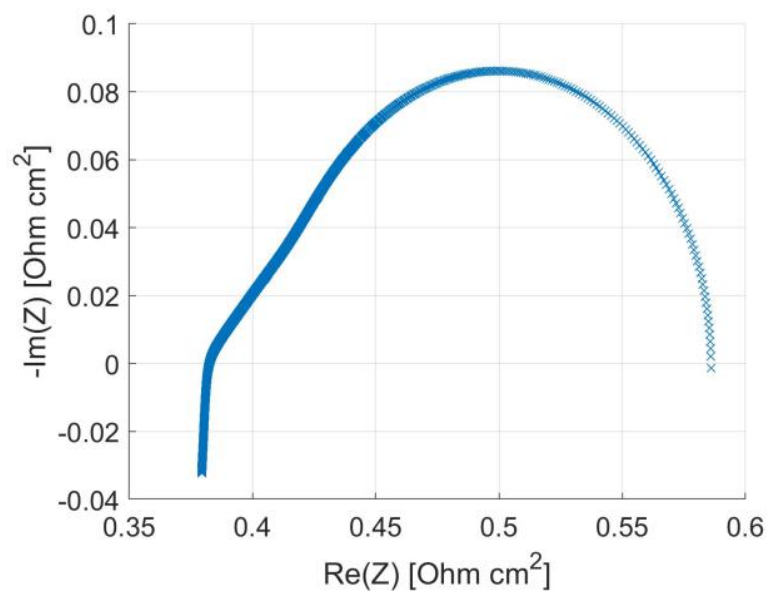

Figure 2: EIS spectra constructed based on the calibration parameter values obtained from calibrating on experimental data in time domain.

\section{Conclusions}

This paper presents, to the best of authors knowledge, for the first time modelling framework of twin analytical ansatzes for modelling PEMFC response in time and frequency domains. Furthermore, due to thermodynamically consistent modelling basis of the twin models, relying on the TC FC electrochemical model with state of the art extrapolation capabilities and replication of the experimental data with one set of 
calibration parameters derived in detail in (2), they are valid in all current density regions. Newly derived frequency domain equivalent of the FC model is based on the analytical solution of PEMFC impedance. Furthermore, the contribution delivers a novel methodology for parametrization of the modeling framework from either of the two separate measurements in time and frequency domains on the other or both at the same time. Consistent combination of these two domains could also enable a significant reduction in the experimental effort. Innovative hybrid methodology of the measurements in time and frequency domains could thus be used for achieving reduced costs and efforts in initial parametrization and during operation in re-parametrization procedures of the modelling framework for virtual observers. It therefore represents a powerful tool for future FC control and diagnostics systems.

\section{Acknowledgments}

The research is partially funded by the Slovenian Research Agency (research core funding No. P2-0401) and by the CD Laboratory for Innovative Control and Monitoring of Automotive Powertrain Systems. 


\section{References}

(1) A. A. Kulikovsky, J. Electrochem. Soc., 161, F263, ( 2014), https://doi.org/10.1149/2.028403jes

(2) A. Kravos, D. Ritzberger, G. Tavčar, C. Hametner, S. Jakubek, and T. Katrašnik, Journal of Power Sources, 454, 227930, (2020), https://doi.org/10.1016/j.jpowsour.2020.227930

(3) W. Gu, D. R. Baker, Y. Liu, and H. A. Gasteiger, Proton exchange membrane fuel cell (PEMFC) down-the-channel performance model, in: Handbook of Fuel Cells, American Cancer Society, 2010. doi:10.1002/9780470974001.f500044.

(4) A. Kravos, D. Ritzberger, G. Tavčar, C. Hametner, S. Jakubek, and T. Katrašnik, EFC19, 8th European Fuel Cell, Piero Lunghi Conference, Naples, 9-11 December, 2019. ENEA 303-304, (2019), https://www.europeanfuelcell.it/media/book_of_proceedings_EFC19.pdf

(5) R. Bellman, R. Corporation, K. M. R. Collection, Dynamic Programming, Rand Corporation research study, Princeton University Press, 1957.

(6) Caisheng Wang, M. H. Nehrir and S. R. Shaw, IEEE Transactions on Energy Conversion, 20, no. 2, pp. 442-451, (2005), doi: 10.1109/TEC.2004.842357.

(7) A. M. Dhirde, N. V. Dale, H. Salehfar, M. D. Mann, and T. Han, IEEE Transactions on Energy Conversion, 25, no. 3, pp. 778-786, (2010), doi:10.1109/TEC.2010.2049267.

(8) I. Pivac, B. Šimić and F. Barbir, Journal of Power Sources, 365, 240-248, ISSN 03787753, (2017), https://doi.org/10.1016/j.jpowsour.2017.08.087.

(9) M Eikerling, A.A Kornyshev, Journal of Electroanalytical Chemistry, 475, Issue 2, Pages 107-123, ISSN 1572-6657, (1999), https://doi.org/10.1016/S0022-0728(99)00335$\underline{6}$.

(10) Seyed Mohammad Rezaei Niya, Ryan K. Phillips, and Mina Hoorfar, Electrochimica Acta, 191, Pages 594-605, ISSN 0013-4686, (2016), https://doi.org/10.1016/j.electacta.2016.01.128.

(11) A.A. Kulikovsky, One-dimensional impedance of the cathode side of a PEM fuel cell: exact analytical solution, J. Electrochem. Soc. 162 (2015) F217-F222, https://doi.org/10.1149/2.0151503jes.

(12) M. Eikerling, A.A. Kornyshev, 453, 1-2, Pages 89-106, ISSN 1572-6657, (1998), https://doi.org/10.1016/S0022-0728(98)00214-9.

(13) M.L. Perry, J. Newman, E.J. Cairns, J. Electrochem. Soc., 145, 5-15, (1998)

(14) A.A. Kulikovsky, Electrochimica Acta, 55, Issue 22, Pages 6391-6401, ISSN 00134686, 2010, https://doi.org/10.1016/j.electacta.2010.06.053. 